Pacific Journal of Mathematics

WEAK COMPACTNESS IN SPACES OF BOCHNER
INTEGRABLE FUNCTIONS AND THE RADON-NIKODÝ

Massif A. Ghoussoub and Paulette SaAb 


\title{
WEAK COMPACTNESS IN SPACES OF BOCHNER INTEGRABLE FUNCTIONS AND THE RADON-NIKODYM PROPERTY
}

\author{
N. GHOUSSOUB AND P. SAAB
}

\begin{abstract}
We characterize Banach spaces $E$ such that $E$ and $E^{*}$ have the Radon-Nikodym property in terms of relatively weakly compact sets of $L^{1}[\lambda, E]$.
\end{abstract}

Introduction. It is well known [1] that if $(\Omega, \Sigma, \lambda)$ is a finite measure space and $E$ is a Banach space, then a relatively weakly compact subset $K$ of $L^{1}[\lambda, E]$ is bounded, uniformly integrable and for every $B \in \Sigma$, the set $\left\{\int_{B} f d \lambda, f \in K\right\}$ is relatively weakly compact in $E$. Moreover, it was shown in [1] that if the Banach space $E$ and its dual $E^{*}$ have the Radon-Nikodym property, then relatively weakly compact subsets of $L^{1}[\lambda, E]$ are completely characterized by the above three conditions. A question that arises naturally is the following: Are the conditions on $E$ and $E^{*}$ to have the Radon-Nikodym property necessary in order that relatively weakly compact subsets of $L^{1}[\lambda, E]$ be exactly those bounded, uniformly integrable subsets $K$ such that for any $B \in \Sigma$, the set $\left\{\int_{B} f d \lambda, f \in K\right\}$ is relatively weakly compact in $E$ ? In [1], it was shown that the condition on $E$ to have the Radon-Nikodym property is indeed necessary. The object of this paper is to show that the condition on $E^{*}$ to have the Radon-Nikodym property is also necessary. This gives a new characterization of Banach spaces $E$ such that $E$ and $E^{*}$ have the Radon-Nikodym property. We also study bounded linear operators $T$ between Banach spaces such that $T$ and its adjoint $T^{*}$ are strong Radon-Nikodym operators.

\section{Definitions and Preliminaries.}

Definition 1. A closed bounded convex subset $C$ of a Banach space $E$ is a Radon-Nikodym (R.N.P) set if for every finite measure space $(\Omega, \Sigma, \lambda)$ and any vector measure $G: \Sigma \rightarrow E$ such that the set $\{G(B) / \lambda(B)$, $B \in \Sigma, \lambda(B)>0\}$ is contained in $C$, there exists a Bochner integrable Radon-Nikodym derivative $f: \Omega \rightarrow C$ such that $G(B)=\int_{B} f d \lambda$, for every $B \in \Sigma$.

For more on (R.N.P) sets see [3] and [4]. 
Definition 2. A bounded linear operator $T$ from a Banach $E$ into a Banach space $F$ is called a strong Radon-Nikodym operator if the closure of $\{T x, x \in E,\|x\| \leq 1\}$ is an (R.N.P) set in $E$.

Accordingly, a Banach space $E$ has the Radon-Nikodym property (R.N.P) iff its closed unit ball is an (R.N.P) set or equivalently if the identity operator on $E$ is a strong Radon-Nikodym operator.

If $T: E \rightarrow F$ is a strong Radon-Nikodym operator then $T$ is an (R.N.P) operator see [2] i.e., for every vector measure $G: \Sigma \rightarrow E$ with $\|G(B)\| \leq \lambda(B)$ for all $B \in \Sigma$, there exists a Bochner integrable function $f: \Omega \rightarrow F$ such that $T G(B)=\int_{B} f d \lambda$ for all $B \in \Sigma$. The converse is not true as any quotient map $Q$ from $l^{1}$ onto $c_{0}$ is an (R.N.P) operator but is not a strong Radon-Nikodym operator. But it follows from [4] if $T: E \rightarrow F$ is a bounded linear operator, then its adjoint $T^{*}$ is a strong Radon-Nikodym operator if and only if $T^{*}$ is an (R.N.P) operator.

Finally, given a finite measure space $(\Omega, \Sigma, \lambda) E$ and $F$ two Banach spaces and $T: E \rightarrow F$ a bounded linear operator, we shall denote by $\tilde{T}$ the natural extension of $T$ to a bounded linear operator from $L^{1}[\lambda, E]$ to $L^{1}[\lambda, F]$.

For all undefined statements and notations we refer the reader to [1].

The following theorem extends the result of $[1, \mathrm{p} .101]$ to operators $T$ : $E \rightarrow F$ such that $T$ and $T^{*}$ are strong Radon-Nikodym operators.

Theorem 1. Let $E$ and $F$ be two Banach spaces and let $T: E \rightarrow F$ be a bounded linear operator such that $T$ and $T^{*}$ are strong Radon-Nikodym operators. Then for any finite measure space $(\Omega, \Sigma, \lambda)$, the operator $\tilde{T}$ : $L^{1}[\lambda, E] \rightarrow L^{1}[\lambda, F]$ sends into relatively weakly compact subsets of $L^{1}[\lambda, F]$ any bounded, uniformly integrable subsets $K$ of $L^{1}[\lambda, E]$ such that for every $B \in \Sigma$ the set $\left\{\int_{B} f d \lambda, f \in K\right\}$ is relatively weakly compact in $E$.

Proof. Let $T: E \rightarrow F$ be a bounded linear operator such that $T$ and $T^{*}$ are strong Radon-Nikdoym operators. Let $(\Omega, \Sigma, \lambda)$ be a finite measure space and let $K \subseteq L^{1}[\lambda, E]$ be a bounded and uniformly integrable subset of $L^{1}[\lambda, E]$ such that for any $B \in \Sigma$ the set $\left\{\int_{B} f d \lambda, f \in K\right\}$ is relatively weakly compact in $E$. Let $\left(f_{n}\right)_{n}$ be a sequence in $K$. Proceed now as in [1, p. 101] to get a countably generated $\sigma$-field $\Sigma_{1}$, such that each $f_{n}$ is measurable with respect to $\Sigma_{1}$, find a subsequence $\left(f_{n_{k}}\right)_{k}$ of $\left(f_{n}\right)_{n}$ and define a countably additive vector measure $G: \Sigma_{1} \rightarrow E$ of bounded variation by

$$
G(B)=\underset{k}{\text { weak }} \operatorname{limit}_{B} f_{n_{k}} d \lambda, \quad \text { for every } B \in \Sigma_{1} \text {. }
$$


Since $T^{*}$ is a Radon-Nikodym operator, it follows from [4] that there exist a Banach space $Z$, such that $Z^{*}$ has RNP, and bounded linear operators $T_{1}: E \rightarrow Z$ and $T_{2}: Z \rightarrow F$ such that the following diagram commutes

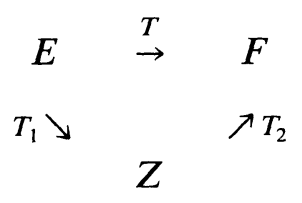

Case 1. Assume that for some $\alpha>0\|G(B)\| \leq \alpha \lambda(B)$, for all $B \in \Sigma_{1}$. It follows that the set $\left\{T_{1} G(B) / \lambda(B), \lambda(B)>0, B \in \Sigma_{1}\right\}$ is contained in the closure $C$ in $Z$ of the set $\left\{T_{1} x, x \in E,\|x\| \leq \alpha\right\}$. But a glance at the construction of [4] reveals that the set $C$ is affinely homeomorphic to the closure in $F$ of the set $\{T x, x \in E,\|x\| \leq \alpha\}$, and one can show that the set $C$ is an R.N.P. set. Therefore there exists a Bochner integrable function $h: \Omega \rightarrow C$ such that

$$
T_{1} G(B)=\int_{B} h d \lambda, \quad \text { for all } B \in \Sigma_{1}
$$

Moreover since $Z^{*}$ has R.N.P and since $\left(\int_{B} T_{1} f_{n_{k}} d \lambda\right)_{k}$ converges weakly to $\int_{B} h d \lambda$ in $Z$ for every $B \in \Sigma_{1}$, it follows that the sequence $\left(\tilde{T}_{1} f_{n_{k}}\right)_{k}$ converges weakly to $h$ in $L^{1}\left[\Sigma_{1}, \lambda, Z\right]$, thus $\left(\tilde{T} f_{n_{k}}\right)_{k}$ converges weakly to $\tilde{T}_{2} h$ in $L^{1}\left[\Sigma_{1}, \lambda, F\right]$, and hence in $L^{1}[\lambda, F]$. An appeal to Eberlein's theorem shows that $\{\tilde{T} f, f \in K\}$ is relatively weakly compact in $L^{1}[\lambda, F]$ and completes the proof of Case 1 .

General case. Let $\left(\Omega_{m}\right)_{m}$ be a partition of $\Omega$ of elements of $\Sigma_{1}$ and such that

$$
\|G(B)\| \leq m \lambda(B)
$$

for all elements $B$ of $\Sigma_{1}$ contained in $\Omega_{m}$. By restricting the sequence $\left(f_{n_{k}}\right)_{k}$ to each of the sets $\Omega_{m}$, by Case 1 , and by an appropriate diagonal process, one can produce a subsequence $\left(h_{j}\right)_{j}$ of $\left(f_{n_{k}}\right)_{k}$ and a sequence $\left(g_{m}\right)_{m}$ of Bochner integrable functions $g_{m}: \Omega_{m} \rightarrow F$ such that:

(i) the sequence $\left(\tilde{T} h_{j \mid \Omega_{m}}\right)_{m}$ converges weakly to $g_{m}$ in $L^{1}\left[\Omega_{m}, \lambda, F\right]$,

(ii) $T G\left(B \cap \Omega_{m}\right)=\int_{B \cap \Omega_{m}} g_{m} d \lambda$, for $B \in \Sigma_{1}$.

Let $g: \Omega \rightarrow F$ be defined as follows:

$$
g(w)=g_{m}(w) \text { if } w \in \Omega_{m} .
$$


It is clear that $g \in L^{\mathrm{l}}[\lambda, F]$ for $g$ is obviously measurable and it follows from (ii) that

$$
\int_{\Omega}\|g(w)\| d \lambda \leq \sum_{m}|T G|\left(\Omega_{m}\right)=|T G|(\Omega)<\infty .
$$

The proof will be complete when we show that the sequence $\left(\tilde{T} h_{j}\right)_{j}$ converges weakly to $g$ in $L^{1}[\lambda, F]$. For this let $L \in\left(L^{1}[\lambda, F]\right)^{*}$. For each $m \geq 1$ let $L_{m}$ be the restriction of $L$ to $L^{1}\left[\Omega_{m}, \lambda, F\right]$. For every $m \geq 1$ we have

$$
\left|L\left(\tilde{T} h_{J}-g\right)\right| \leq\left|\sum_{l=1}^{m} L_{l}\left(\tilde{T} h_{j \mid \Omega_{t}}-g_{i}\right)\right|+\|L\| \int_{U \Omega_{i} ; i>m}\left\|\tilde{T} h_{i}-g\right\| d \lambda .
$$

Since the sequence $\left(h_{j}\right)_{j}$ is uniformly integrable, there exists $m \geq 1$ such that $\int_{U \Omega_{i} ; i \geq m}\left\|\tilde{T} h_{j}-g\right\| d \lambda$ is arbitrary small for all $j \geq 1$. Since $\tilde{T} h_{j \mid \Omega_{t}}$ converges weakly to $g_{l}$, it follows that $\left|\sum_{i=1}^{m} L\left(\tilde{T} h_{\mid, \Omega_{l}}-g_{l}\right)\right|$ is arbitrary small as $j \rightarrow \infty$. Hence $L(\tilde{T} h,-g) \rightarrow 0$ as $j \rightarrow \infty$. This completes the proof.

The following proposition establishes the fact that if $T^{*}$ fails to be a strong Radon-Nikodym operator, then the conclusion of Theorem 1 is no more valid.

Proposition. If $T$ is a bounded linear operator from a Banach space $E$ into a Banach space $F$ such that $T^{*}$ fails to be a strong Radon-Nikodym operator, then there exists a finite measure space $(\Omega, \Sigma, \lambda)$, a bounded uniformly integrable subset $K$ of $L^{1}[\lambda, E]$ such that the set $\left\{\int_{B} f d \lambda, f \in K\right\}$ is relatively weakly compact in $E$ for any $B \in \Sigma$, but the set $\{\tilde{T} f, f \in K\}$ is not relatively weakly compact in $L^{1}[\lambda, F]$.

Proof. Suppose that $T^{*}$ fails to be a strong Radon-Nikodym operator. Let $\Delta=\{-1,1\}^{\mathbf{N}}$ denote the Cantor group with Haar measure $m$ and let $\left\{\Delta_{n, l}, 1 \leq i \leq 2^{n}\right\}$ denote the standard $n$th partition of $\Delta$ with $\Delta_{01}=\Delta$, $\Delta_{n, i}=\Delta_{n+1,2 l-1} \cup \Delta_{n+1,2 l}, \Delta_{n, i}$ is clopen, and $m\left(\Delta_{n, i}\right)=1 / 2^{n}$. It follows from the dichotomy theorem of Stegall [4] that the operator $T$ must factor the Haar operator $H: l^{1} \rightarrow L_{\infty}(m)$ which takes the basis of $l^{1}$ into the usual Haar basis of $C(\Delta)$ considered as a subspace of $L_{\infty}(m)$. Indeed the Haar operator is defined as follows:

$$
\text { if } h_{n l}=\chi_{\Delta_{n+1,2 l-1}}-\chi_{\Delta_{n+1,2 l}}, \quad n \geq 0,1 \leq i \leq 2^{n} \text { then } H e_{n l}=h_{n i},
$$

here $\left\{e_{n l}, n \geq 0,1 \leq i \leq 2^{n}\right\}$ is an enumeration of the usual $l^{1}$ basis. Let $U: l^{1} \rightarrow E$ and $V: F \rightarrow L_{\infty}(m)$ be bounded linear operators such that $H=V \circ T \circ U$ as illustrated in the following diagram. 


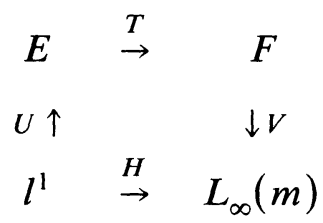

Consider the following sequence $\left(f_{n}\right)_{n}$ in $L^{1}\left[m, l^{1}\right]$ with

$$
f_{n}(t)=\frac{1}{n} \sum_{j=1}^{n} \sum_{i=1}^{2^{j}} h_{j i}(t) e_{j i}, \quad \text { for } t \in \Delta .
$$

The sequence $\left(f_{n}\right)_{n}$ is easily seen [2] to have the following properties

(i) $\sup _{n}\left\|f_{n}(t)\right\|=1$ m.a.e.

(ii) $\sup _{\left\|x^{*}\right\| \leq 1} \int\left|x^{*} \circ f_{n}\right| d m$ approaches zero as $n \rightarrow \infty$.

It follows that for every Borel $B$ set in $\Delta$ the sequence $\left(\left\|\int_{B} f_{n} d m\right\|\right)_{n}$ approaches zero as $n \rightarrow \infty$. The sequence $\left(f_{n}\right)_{n}$ is bounded and uniformly integrable in $L^{1}\left[m, l^{1}\right]$ and $\left(\int_{B} f n d m\right)_{n}$ is a null sequence in $l^{1}$. We claim that the sequence $\left(\tilde{H} f_{n}\right)$ is not relatively weakly compact in $L^{1}\left[m, L_{\infty}(m)\right]$. For this note that for each $n \geq 1$

$$
\tilde{H} f_{n}(t)=\frac{1}{n} \sum_{j=1}^{n} \sum_{i=1}^{2^{\prime}} h_{j i}(t) h_{j l}, \quad t \in \Delta ;
$$

therefore $\tilde{H} f_{n}(t)$ takes its values in $C(\Delta)$, to prove the claim all we need to show is that $\left(\tilde{H} f_{n}\right)_{n}$ is not relatively weakly compact in $L^{1}[m, C(\Delta)]$. To this end note that since for every Borel set $B$ the sequence $\left(\int_{B} \tilde{H} f_{n} d m\right)_{n}$ converges to zero in $C(\Delta)$, it follows that every weakly convergent subsequence of $\left(\tilde{H} f_{n}\right)_{n}$ in $L^{1}[m, C(\Delta)]$ must converge to zero. Let $L \in$ $\left(L^{1}[m, C(\Delta)]\right)^{*}$ be defined as follows: for $\psi \in L^{1}[m, C(\Delta)]$

$$
L(\psi)=\int_{\Delta} \psi(t)(t) d m
$$

then

$$
L\left(\tilde{H} f_{n}\right)=\frac{1}{n} \int_{\Delta} \sum_{j=1}^{n} \sum_{i=1}^{2^{J}} h_{j i}(t) h_{j i}(t) d m=1 .
$$

This shows that the sequence $\left(\tilde{H} f_{n}\right)_{n}$ has no weakly convergent subsequence in $L^{1}[m, C(\Delta)]$. The sequence $\left(\tilde{U} f_{n}\right)_{n}$ is bounded and uniformly integrable in $L^{1}[m, E]$ and the set $\left\{\int_{B} \tilde{U} f_{n} d \lambda, n \geq 1\right\}$ is relatively weakly compact in $E$ for all Borel sets $B$ of $\Delta$, yet since $T$ factors the Haar operator $H$, the sequence $\left(\tilde{T} \tilde{U} f_{n}\right)_{n}$ cannot have a weakly convergent subsequence in $L^{1}[m, F]$. This completes the proof. 
Corollary 3. A Banach space $E$ and its dual $E^{*}$ have (R.N.P) if and only if for every finite measure space $(\Omega, \Sigma, \lambda)$, any bounded and uniformly integrable subset $K$ of $L^{1}[\lambda, E]$ is relatively weakly compact whenever for every $B \in \Sigma$, the set $\left\{\int_{B} f d \lambda, f \in K\right\}$ is relatively weakly compact in $E$.

\section{REFERENCES}

[1] J. Diestel and J. J. Uhl, Jr. Vector measures, Mathematical Survey No. 15, A.M.S., Providence, 1977.

[2] G. A. Edgar, Asplund operators and A. E. convergences, J. Multivariate Anal., 10 (1980), 460-466.

[3] E. Saab, A characterization of $w^{*}$-compact convex sets having the Radon-Nikodym property, Bull. Sci. Math., (2), 104 (1980), 79-88.

[4] C. Stegall, The Radon-Nikodym property in conjugate Banach spaces, II, Trans. Amer. Math. Soc., 264 (1981), 507-519.

Received February 22, 1982.

THE UNIVERSITY OF BRITISH COLUMBIA

VANCouver, B.C., Canada V6T 1Y4

AND

THE UNIVERSITY OF MisSOURI-COLUMBIA

Columbia, MO 65211 


\section{PACIFIC JOURNAL OF MATHEMATICS \\ EDITORS}

DONALD BABBITT (Managing Editor)

University of California

Los Angeles, CA 90024

Hugo RossI

University of Utah

Salt Lake City, UT 84112

C. C. MOOre and ARThur OGus

University of California

Berkeley, CA 94720
J. Dugundi

Department of Mathematics

University of Southern California

Los Angeles, CA 90089-1113

R. FINN and H. SAMELSON

Stanford University

Stanford, CA 94305

ASSOCIATE EDITORS

$\begin{array}{lllll}\text { R. Arens } & \text { E. F. Beckenbach } & \text { B. H. Neumann } & \text { F. Wolf } & \text { K. Yoshida }\end{array}$ $(1906-1982)$

\section{SUPPORTING INSTITUTIONS}

UNIVERSITY OF ARIZONA

UNIVERSITY OF BRITISH COLUMBIA

CALIFORNIA INSTITUTE OF TECHNOLOGY

UNIVERSITY OF CALIFORNIA

MONTANA STATE UNIVERSITY

UNIVERSITY OF NEVADA, RENO

NEW MEXICO STATE UNIVERSITY

OREGON STATE UNIVERSITY
UNIVERSITY OF OREGON

UNIVERSITY OF SOUTHERN CALIFORNIA

STANFORD UNIVERSITY

UNIVERSITY OF HAWAII

UNIVERSITY OF TOKYO

UNIVERSITY OF UTAH

WASHINGTON STATE UNIVERSITY

UNIVERSITY OF WASHINGTON 


\section{Pacific Journal of Mathematics}

\section{Vol. 110, No. $1 \quad$ September, 1984}

Wojciech Abramczuk, A class of surjective convolution operators $\ldots \ldots \ldots \ldots 1$

K. Adachi, Extending bounded holomorphic functions from certain

subvarieties of a weakly pseudoconvex domain $\ldots \ldots \ldots \ldots \ldots \ldots$

Malvina Florica Baica, An algorithm in a complex field and its application

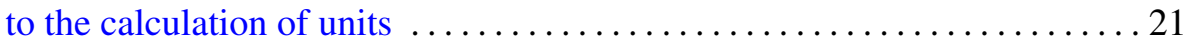

Giuliana Bianchi and Robert Cori, Colorings of hypermaps and a

conjecture of Brenner and Lyndon $\ldots \ldots \ldots \ldots \ldots \ldots \ldots \ldots \ldots \ldots \ldots \ldots$

Ronald James Evans, Determinations of Jacobsthal sums . . .......... 49

Leslie Foged, Characterizations of $\aleph$-spaces .................... 59

Nassif A. Ghoussoub and Paulette Saab, Weak compactness in spaces of

Bochner integrable functions and the Radon-Nikodým property . . . . . . 65

J. Gómez Gil, On local convexity of bounded weak topologies on Banach

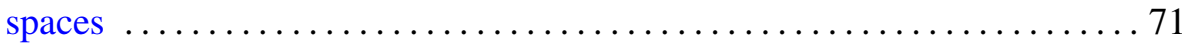

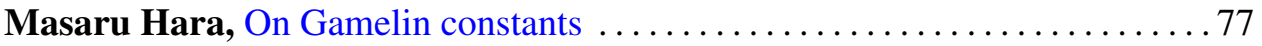

Wilfried Hauenschild, Eberhard Kaniuth and Ajay Kumar, Harmonic

analysis on central hypergroups and induced representations $\ldots \ldots \ldots 83$

Eugenio Hernandez, An interpolation theorem for analytic families of

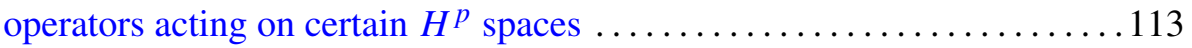

Thomas Alan Keagy, On "Tauberian theorems via block-dominated

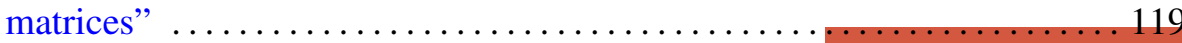

Thomas Landes, Permanence properties of normal structure $\ldots \ldots \ldots \ldots \ldots 125$

Daniel Henry Luecking, Closed ranged restriction operators on weighted

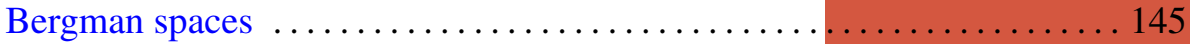

Albert Thomas Lundell, The $p$-equivalence of $\mathrm{SO}(2 n+1)$ and $\mathrm{Sp}(n) \ldots \ldots 161$

Mark D. Meyerson, Remarks on Fenn's "the table theorem" and Zaks' "the chair theorem" ..................................... 167

Marvin Victor Mielke, Homotopically trivial toposes . . . . . . . . . . 171

Gerard J. Murphy, Hyperinvariant subspaces and the topology on Lat A . . 183

Subhashis Nag, On the holomorphy of maps from a complex to a real manifold

Edgar Milan Palmer and Robert William Robinson, Enumeration of self-dual configurations ................................. 203

John J. Walsh and David Clifford Wilson, Continuous decompositions

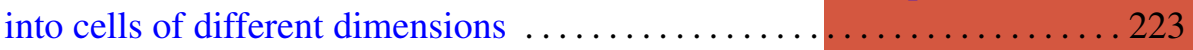

Walter John Whiteley, Infinitesimal motions of a bipartite framework .....233 